\title{
The radial velocities and physical parameters of ER Vul ${ }^{\star} \star \star$
}

\author{
R. Duemmler ${ }^{1,2}$, C. Doucet ${ }^{1,3}$, F. Formanek ${ }^{1,4}$, I. Ilyin $^{1}$, and I. Tuominen ${ }^{1}$ \\ 1 Astronomy Division, PO Box 3000, 90014 University of Oulu, Finland \\ 2 Present address: Wilhelm-Leuschner-Str. 16, 60329 Frankfurt/M., Germany \\ 3 Magistère de Physique Fondamentale, Université Paris XI, 91405 Orsay, France \\ ${ }^{4}$ Laboratoire d'Optique Physique ESPCI, 10 rue Vauquelin, 75005 Paris, France
}

Received 26 July 2002 / Accepted 21 February 2003

\begin{abstract}
ER Vul is an eclipsing binary consisting of two solar type stars in a very close orbit with a period of 0.7 . Accordingly, the two stars rotate very fast, leading to a blending of many spectral features at all phases. Therefore, measuring the radial velocity curve without systematic errors is not trivial. Here, we use a two-dimensional cross-correlation method applied to $137 \mathrm{high}$ quality spectra, collected over 3 years, in order to obtain the radial velocity curve and determine the orbital and some physical parameters of the system from it. Primarily, we improve the binary period to $0.69809458 \pm 0 \mathrm{~d} 00000014$, and find that the two amplitudes are slightly smaller than those measured by others, while the mass ratio is still similar. While at least the primary almost fills its Roche lobe, the system is still detached, i.e. not yet a fully fledged W UMa-system. The behaviour of the Ca II IRT line at $8662 \AA$ confirms that the secondary is the more active component, and that the chromospheric emission is not symmetrically distributed over the surfaces of either star.
\end{abstract}

Key words. stars: individual: ER Vul - binaries: spectroscopic - binaries: eclipsing - stars: late-type - techniques: radial velocities

\section{Introduction}

ER Vul (HD 200391) is an eclipsing, double-lined spectroscopic binary which consists of two nearly identical, solar-type stars in a very close orbit, the period being close to 0.7 .

For eclipsing, double-lined binaries, much can be said about the physical parameters of the individual components from an analysis of the light and radial velocity curves. Thus, these systems have always been very interesting for astrophysicists. However, it is important that there are no systematic errors which would influence the physical parameters.

ER Vul has very broad lines in its spectrum. This makes radial velocity $(R V)$ measurements very inaccurate. Furthermore, since both stars are of similar spectral type, i.e. the same lines occur and the widths are the same, even at phases of maximal separation many lines of the two stars are blended. It happens

Send offprint requests to: I. Ilyin, e-mail: ilya.ilyin@oulu. fi

* Based on observations made with the SOFIN échelle spectrograph at the Nordic Optical Telescope, operated on the island of La Palma jointly by Denmark, Finland, Iceland, Norway, and Sweden, in the Spanish Observatorio del Roque de los Muchachos of the Instituto Astrofisica de Canarias; and the coudé spectrograph at the $2 \mathrm{~m}$-RCC-telescope of the National Astronomical Observatory at Rozhen, Bulgaria.

$\star \star$ Full Table 1 is only available in electronic form at the CDS via anonymous ftp to cdsarc.u-strasbg.fr $(130.79 .128 .5)$ or via http://cdsweb.u-strasbg.fr/cgi-bin/qcat?]/A+A/402/745 often that a certain line of one star, redshifted by its orbital velocity, and a different line at longer wavelengths of the other star, blueshifted by its orbital velocity, become blended (Fig. 1 shows an example near $6440 \AA$ ), so that several features cannot be uniquely assigned to one star. These effects lead to systematic errors in $R V$ measurements.

Another effect is the stellar activity. Hall (1976) classified ER Vul as a short-period RS CVn system. Both components are very active and show large spots (both cool and hot). These spots will deform the line profiles (this is how the presence of the spots is found and how surface imaging is able to invert the spectra into maps of the surface distribution of temperature), leading to systematic errors in the $R V \mathrm{~s}$ measured from them. Since in ER Vul the rotation and the orbital periods are very likely the same, the distortions will have a certain dependence on orbital phase, depending on the longitudes of the spots. Therefore, the $R V$ curve may be deformed. A solution to this problem is to measure the $R V$ curve over a long time span; the limited lifetime of the spots and/or their movement, e.g. due to differential rotation, will then change the deformation of the curve with time and it can be hoped that the distortion of the single $R V$ will average out over all the data.

Several $R V$ curve solutions for ER Vul have already been published. The first was given by Northcott \& Bakos (1967). They do not describe in detail their procedure for the $R V$ measurements; their curve is, however, comparable to those of 
later works. McLean (1982) obtained a few $R V$ s with a crosscorrelation technique using a $R V$ standard as template, i.e. neglecting the blending effects due to the much higher rotational velocities of the ER Vul components and the blending between the two components of each feature. Due to this and the limited number of $R V \mathrm{~s}$ available, the errors of the parameters are large. The latest investigation is that of Hill et al. (1990), who also worked with cross-correlation. They took into account the rotational broadening, still using only a single template, i.e. the cross-correlation peaks are double-peaked. The peaks were then approximated by Gaussians to obtain the $R V$. The method may be sufficiently accurate when the two stars are near maximum separation, but it will more and more fail the closer the two $R V$ s get to each other, resulting in unknown systematics. Furthermore, the systematic error in the $R V$ depends on the spectral range and the spectral features that are used: when a redshifted feature blends with a blueshifted, different feature from the other binary component the cross-correlation becomes deformed and possibly the position systematically shifted.

Very few $R V \mathrm{~s}$ with large errors from cross-correlation are also presented by Gunn et al. (1996).

We obtained a large number of spectra for the purpose of surface (Doppler-)imaging of ER Vul. Given the possible systematics in earlier investigations we decided to use these spectra to obtain independently a new $R V$ curve and derive some constraints on physical parameters from it.

\section{Observations and reductions}

The observations consist of 130 échelle spectra obtained with the high-resolution échelle spectrograph SOFIN (Tuominen et al. 1999; Ilyin 2000) mounted at the $2.56 \mathrm{~m}$ Nordic Optical Telescope (NOT) in La Palma, Spain. These spectra were obtained with the medium resolution camera $(R \approx$ 70000 ), consisting of typically 17 orders of about $50 \AA$ length. Additionally, 7 spectra from the coudé spectrograph at the $2 \mathrm{~m}$-RCC-telescope at the National Astronomical Observatory at Rozhen, Bulgaria, are available. They are centred around $6430 \AA$ and have a length of about $60 \AA$. The dates of the observations are given in Table 1. The spectra cover the time interval June 1993-August 1996. In the order around $6440 \AA$, the typical signal-to-noise ratios are within 150-300.

The reduction of all observations has been done with the échelle spectrum reduction package 4A (Ilyin 2000); the basic steps are bias subtraction, flatfielding, scattered light subtraction, weighted spectrum extraction and wavelength calibration.

The wavelength calibration is based on the comparison spectrum images consisting emission lines, $\mathrm{Fe}-\mathrm{Ar}$ for the coudé, and Th-Ar for the échelle spectra. For the échelle spectra, all Th-Ar lines in all orders of the comparison images are used in a two-dimensional dispersion curve (for details see Ilyin \& Duemmler 1999). Several orders of the échelle spectra and the red end of the coudé spectra contain telluric atmospheric lines. These are used to correct possible small shifts in the wavelength zero-point. Shifts may occur, caused by bending of the Cassegrain-mounted SOFIN and the changes of ambient conditions, as well as (for both spectrographs) by the slit effect, the variable illumination of the collimator caused by seeing variations and systematical errors in setting and guiding of the stellar image. After the wavelength calibration, the spectra are transformed into heliocentric wavelengths.

The continuum normalization is done iteratively. For spectra obtained close to the conjunctions, the comparison to an artificially spun-up solar spectrum is used to define the continuum level, described by a polynomial of low degree. Then, for spectra at slightly different phases, already normalized spectra are available to define the continuum level, and so on. Thus, in the end, the spectra have the same continuum level as the original (not spun-up) sun, i.e. the effect of lowering the continuum level due to blending of the very broad features is automatically taken into account.

It is worth mentioning that $4 \mathrm{~A}$ estimates the errors of each pixel in the original image (after bias subtraction) assuming Poissonian noise and propagates these errors through each step of the reduction procedure, including the calibration images, e.g. during the flatfield correction. In this way, reliable errors are available for each pixel in the final spectrum in both flux and wavelength resulting also in individual errors for each $R V$.

\section{The radial velocities}

\subsection{The construction of the templates}

Both components of ER Vul are nearly solar-type stars. The solar spectrum would therefore make a good template for crosscorrelation $R V$ measurements. The problems are the large rotational velocities and the composite spectra of two stars with similar luminosities. The first step, therefore, is to determine the projected rotational velocities $v \sin i$ for both stars.

Spectra close to the two eclipses were identified from earlier solutions of the binary parameters. For each eclipse, these spectra were averaged in order to reduce the noise in the eclipse spectra. If the eclipses were total, each of these two average spectra represent the spectrum of one of the two stars alone.

Solar spectra were extracted from the solar FTS spectrum (Kurucz et al. 1984) for the six selected spectral orders free from telluric lines and with suitable lines of the two stellar components. Using a linear limb-darkening approximation and the limb-darkening coefficients interpolated from the tables of Al-Naimiy (1978), these solar spectra were artificially spun-up (convolved with the rotation profile as given by Gray 1992, his Eq. (17.12)) to different projected rotational velocities $v \sin i$.

Cross-correlations of all these spectra with the average spectra at the two eclipse phases were performed and the optimal $v \sin i$ identified by maximizing the cross-correlation peak height. The resulting value is the weighted average of the velocity derived from each of the spectral orders used with the weight defined by the quality of the cross-correlation. The results are: $v \sin i(\operatorname{star} 1)=(97.4 \pm 1.3) \mathrm{km} \mathrm{s}^{-1}, v \sin i(\operatorname{star} 2)=$ $(96.7 \pm 2.2) \mathrm{km} \mathrm{s}^{-1}$. Thus, the difference of the two projected rotational velocities is $(0.7 \pm 2.6) \mathrm{km} \mathrm{s}^{-1}$, i.e. the two stars have identical rotational velocities. The final value is, therefore, the weighted average of all 8 values at the two phases:

$v \sin i=(97.0 \pm 1.1) \mathrm{km} \mathrm{s}^{-1}$.

However, due to the rather small inclination of the binary system, the eclipses are not total, and as it later turned out (see 

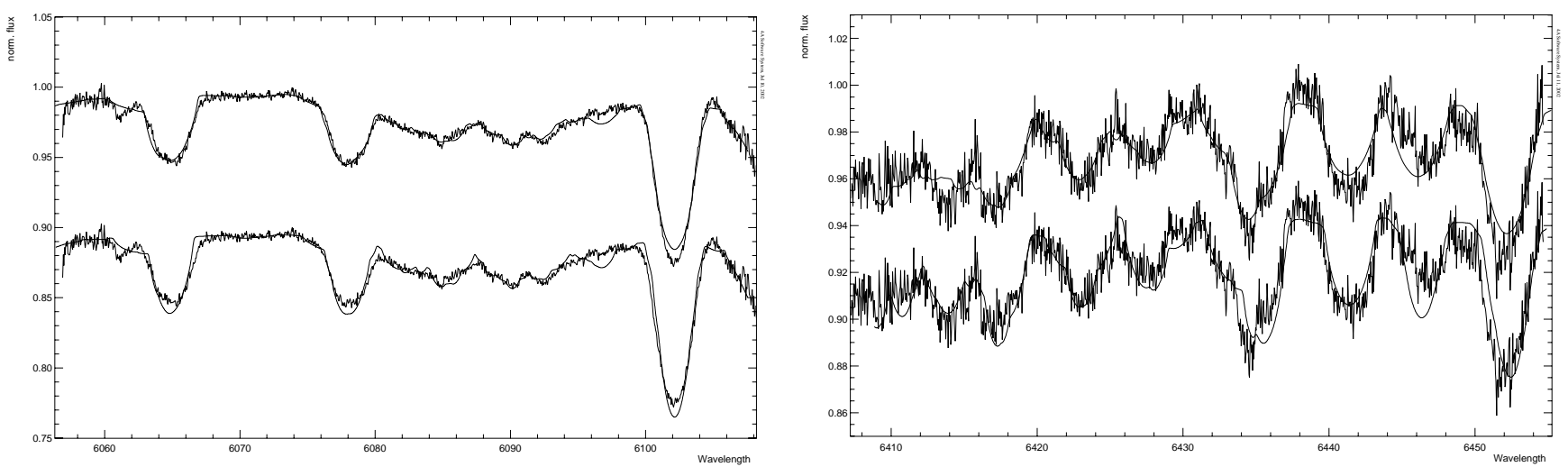

Fig. 1. a) (Left) the average spectrum of ER Vul near one of the conjunction phases compared to the spectrum of the Sun, artificially spun-up to $v \sin i=97 \mathrm{~km} \mathrm{~s}^{-1}$ (top) and to $81 \mathrm{~km} \mathrm{~s}^{-1}$ (bottom). b) (Right) the spectrum of ER Vul near the phase of maximum separation (HJD 2449585.43144) compared to an artificial composite of two solar spectra, shifted to our measured $R V=106.8 \mathrm{~km} \mathrm{~s}^{-1}$ for the red component and $\Delta R V=280 \mathrm{~km} \mathrm{~s}^{-1}$ for the velocity difference between the two components. Again, two templates are shown: both stars spun up to $97 \mathrm{~km} \mathrm{~s}^{-1}$ (top), and the primary spun up to $81 \mathrm{~km} \mathrm{~s}^{-1}$, the secondary to $71 \mathrm{~km} \mathrm{~s}^{-1}$ (bottom). A single spectrum is displayed, not an average of several spectra as in a), so the noise is comparatively large; note also the expanded scale in b) compared to a).

Sect. 4), in both eclipses the same star is measured (the one with the larger $v \sin i$ ).

The result is surprising, given that Hill et al. (1990) obtained significantly smaller values $\left(81 \mathrm{~km} \mathrm{~s}^{-1}\right.$ and $\left.71 \mathrm{~km} \mathrm{~s}^{-1}\right)$. Their values are consistent with the ones given by Huisong \& Xuefu (1987). An attempt has therefore been made to measure $v \sin i$ using the Fourier transform of several features (see e.g. Gray 1992, Ch. 17). Due to the large rotational velocity, there are no unblended lines, and only very few features gave a useful power spectrum. While the minima tend to give slightly smaller values of $v \sin i$ by about $3-5 \mathrm{~km} \mathrm{~s}^{-1}$, with a scatter of about $2 \mathrm{~km} \mathrm{~s}^{-1}$, the value in Eq. (1) is based on much more information (only 2 spectral features gave a total of 3 useful minima in the power spectrum). We, therefore, do not consider this difference significant, and will use the value of Eq. (1) in the following.

We demonstrate that our result for $v \sin i$ may not be perfect, but is certainly better for the representation of our spectra than the values by Hill et al. (1990) in Fig. 1, where we show the spectrum at one of the eclipse phases (the other looks very similar) and a spectrum at a phase close to maximum separation; overplotted are spectra which are composed of two spun-up solar spectra, shifted to the measured $R V$ and $R V$ difference (see below). The spectra are spun-up to our value of $97 \mathrm{~km} \mathrm{~s}^{-1}$ for both stars and to $81 \mathrm{~km} \mathrm{~s}^{-1}$ for the primary and to $71 \mathrm{~km} \mathrm{~s}^{-1}$ for the secondary. It is clear that the values of Hill et al. (1990) are too small. In particular, we call attention to the features at 6435 and $6452 \AA$ : these are both blends of redshifted features of the primary and blueshifted, different features of the secondary. It is clear that our template yields a very good match for the observed features, while the features obtained for the combination of $v \sin i$ values of 81 and $71 \mathrm{~km} \mathrm{~s}^{-1}$ show systematic differences in both shape and position.

We finally remark, however, that the exact value of $v \sin i$ has not a very large influence on the $R V$ at phases near maximum separation. In the spectrum shown in Fig. 1b the velocity measured using the composite with the Hill et al. values is different by $(1 \pm 1) \mathrm{km} \mathrm{s}^{-1}$ from our value. We expect significantly different values at phases near conjunction, where the representation of the blended profiles of the two stars depends sensitively on the broadening. If our $v \sin i$ would be that much wrong, we would expect large and systematic deviations from the $R V$ curve near conjunction with small deviations near the extrema. Later, in Sect. 4, we will get from this another support for our $v \sin i$ at least for the primary.

Finally, a set of template spectra was constructed, each consisting of two solar spectra, each of which is spun up to $97 \mathrm{~km} \mathrm{~s}^{-1}$. One spectrum remains unshifted, the other is shifted by $\Delta R V$ toward the blue; $\Delta R V$ varies from template to template by $10 \mathrm{~km} \mathrm{~s}^{-1}$. The unshifted and shifted spun-up solar spectra are then averaged to give an artificial composite spectrum.

This method to construct templates assumes that the line intensities are equal for the two stellar components. From the light curves, the ratio between the two components in $V$ is from 0.5 (Mennella 1990) to 0.8 (Hill et al. 1990). However, a small difference in $V$ does not imply directly that there will be a difference in the line depth of the two components, and based upon this fact we have to assume that the line profiles are rather similar to these derived from the solar spectrum. Figure 1 shows indeed that the solar spectrum represents rather well the observed spectrum of ER Vul. One other alternative way to construct the stellar templates would be the extraction of line profiles from each of the two stellar components at the maximal separation with the subsequent alignment in radial velocities and averaging. On the other hand, this would limit us with the number of lines which can be extracted because of the blending between the lines. Hence, the use of the solar spectrum was the final choice for the composite template. It is worth to mention that the method of the composite templates is somewhat similar to the TODCOR approach (Zucker \& Mazeh 1994); the main difference is that we do not allow for the adaptive scaling of the line profiles for each component separately, but we do adaptive 
scaling of the whole composite template to the observed spectrum at every lag of the cross-correlation offset.

\subsection{The measurement of the radial velocities}

The first step is the selection of spectral orders suitable for the measurements of $R V \mathrm{~s}$. The spectra should be free of telluric atmospheric lines.

The coudé spectra from Rozhen, of course, consist only of one "order", which covers approximately the wavelength range 6400-6460 $\AA$; a few atmospheric lines are present in the very red end of this range. This region is cut off prior to the measurements. Another selection criterion is signal-to-noise $(S / N)$ ratio. Also, of course, there should be some strong lines included.

For the SOFIN échelle spectra, six selected spectral orders were used for the measurements, where, however, the very bluest order is present only in the data of one observing run. Sometimes, lines are close to the edges of the order or even cut off. This makes the continuum correction uncertain near these edges, leading sometimes to severe profile distortions. If this happens, the line or the distorted part of it is masked out prior to $R V$ measurement.

The two $R V$ s for each order are determined by a crosscorrelation technique. Each order for each image is crosscorrelated with each composite template. Due to the fact that the template is a composite spectrum as well, the crosscorrelation function for the correct template only has a single peak. This peak will be highest for the template which resembles most closely the spectrum of ER Vul at that time. Different from the cross-correlation used e.g. by Hill et al. (1990), the cross-correlation is also able to take into account blends of different lines from different stars (very important in the linerich order covering the wavelength range around 26430). Given that our templates consist of an unshifted solar spectrum plus a blueshifted one, the cross-correlation offset will be $R V_{\text {red }}$, the $R V$ of the redshifted component. The template, which leads to the highest cross-correlation peak, identifies $\Delta R V$, the relative shift between the redshifted and blueshifted components.

Because our templates were created with a step of $10 \mathrm{~km} \mathrm{~s}^{-1}$ in $\Delta R V$, the exact value of $R V_{\text {red }}$ and $\Delta R V$ are obtained by an interpolation. Near the best template, the cross-correlation peak height and $R V_{\text {red }}$ vary smoothly with $\Delta R V$. The cross-correlation peak heights vs. $\Delta R V$ for the four highest cross-correlation peaks are fitted by a parabola, whose maximum position gives the exact value of $\triangle R V$; the fit of a line or, if necessary, of a parabola to $R V_{\text {red }}$ vs. $\Delta R V$ allows to calculate the exact value of $R V_{\text {red }}$ for the exact value of $\Delta R V$.

Each $R V$ is accompanied by an error. The first error source is the accuracy with which the cross-correlation can be performed. This error is given by $4 \mathrm{~A}$ and described in details in Ilyin (2000). The error is calculated from the weighted linear regression fit of the two spectra for the best position of the cross-correlation offset. The error depends upon the residual sum of squares of the linear fit which is a function of variances of individual pixels rebinned for the offset lag, and systematical differences between the two profiles (ER Vul spectrum and the template). The variances of the pixels are initially obtained with the Poissonian statistics and subsequently modified through out the reduction process. Cross-correlation, used to derive the radial velocity, for different orders have different errors. The error is smaller for a better template. We therefore decided to use as the cross-correlation error that of the template closest to the interpolated $\Delta R V$. However, another error source is the quality of the two fits used in the interpolations. These errors are sometimes negligible, but are sometimes the dominant error source, depending on the smoothness of the variation of peak height and $R V_{\text {red }}$ vs. $\Delta R V$ and the distance of the optimal $\Delta R V$ from the nearest template's $\Delta R V$. These errors are added in quadrature. Since $R V_{\text {red }}$ is obtained directly from the interpolation of $R V_{\text {red }}$ vs. $\Delta R V$, while $R V_{\text {blue }}$ is obtained from the interpolated $R V_{\text {red }}$ and the interpolated $\Delta R V, R V_{\text {blue }}$ has the larger error of the two $R V \mathrm{~s}$.

It should also be noted that the two $R V \mathrm{~s}$ are not exactly independent, because an error in $\Delta R V$ will lead to correlated errors in $R V_{\text {red }}$ and $R V_{\text {blue }}$.

$R V_{\text {red }}$ refers to different stars in different halves of the orbit. At phases near conjunction, when two almost identical spectral features are heavily blended, it is impossible to say which feature belongs to which star, without an orbital solution. At phases near maximum $\Delta R V, R V_{1}$ is assigned to the star, whose $R V$ s show the smaller amplitudes. With the so identified $R V_{1}$ and $R V_{2}$, a preliminary orbital fit was done to provide the identification of the stars near the conjunction phases.

The cross-correlation gives up to 6 independent $\left(R V_{1}\right.$, $R V_{2}$ )-pairs from the different orders. The reddest order is dominated by the infrared triplet (IRT) line of Ca II at $8662 \AA$; since this is a line sensitive to chromospheric activity and partially filled in by emission (Gunn \& Doyle 1997), it is kept separate. For the other 5 orders, if present, the radial velocities of the same components were averaged, where a weight was used, which is the inverse variance, based on the errors of the individual measurements. The error of the final $R V_{1}$ or $R V_{2}$ is given by the error of the weighted mean; typically, these errors are $1-3 \mathrm{~km} \mathrm{~s}^{-1}$. The final values of $R V_{1}$ and $R V_{2}$, as well as the values obtained for the order with the Ca II IRT-line, are given, together with their errors, in Table 1 (the full table is only available electronically).

\section{The radial velocity curve}

\subsection{The orbital solutions based on our data}

The radial velocities $R V_{1}, R V_{2}$ are used in a double-lined orbital fit (for some details on the code used see Duemmler et al. 1997). The fit is weighted, i.e. each $R V$ is accompanied by a weight, which is the inverse variance based on the error given in Table 1.

The errors of the fit as well as the derived parameters were determined by 10000 bootstrap runs (see Duemmler et al. 1997 and, e.g., Efron \& Tibshirani 1993). Artificial data sets are created: for each time point of a real measurement, a randomly chosen value taken from the $\mathrm{O}-\mathrm{C}$ values (i.e. residuals) of the real data is added to the $R V$ computed from the orbital solution. Since on average low-weighted $R V \mathrm{~s}$ tend to have larger $\mathrm{O}-\mathrm{C}$, each $\mathrm{O}-\mathrm{C}$ is accompanied by its original weight; the choice of 
Table 1. 2 sample records from the table with the measured radial velocities. The full table (only available electronically at the CDS) consists of 137 records. $R V_{1}, R V_{2}$ are the radial velocities of the primary and the secondary, respectively; $R V^{\mathrm{Ca}}$ is the radial velocity of the Ca II IRT line at $8662 \AA$. Errors of $-999.999 \mathrm{~km} \mathrm{~s}^{-1}$ indicate missing measurements. $R V_{1}, R V_{2}$ are weighted averages of measurements from up to 5 orders.

\begin{tabular}{crrrrrrrr}
\hline \hline HJD & $\begin{array}{r}R V_{1} \\
\left(\mathrm{~km} \mathrm{~s}^{-1}\right)\end{array}$ & $\begin{array}{r}\sigma_{R V_{1}} \\
\left(\mathrm{~km} \mathrm{~s}^{-1}\right)\end{array}$ & $\begin{array}{r}R V_{2} \\
\left(\mathrm{~km} \mathrm{~s}^{-1}\right)\end{array}$ & $\begin{array}{r}\sigma_{R V_{2}} \\
\left(\mathrm{~km} \mathrm{~s}^{-1}\right)\end{array}$ & $\begin{array}{r}R V_{1}^{\mathrm{Ca}} \\
\left(\mathrm{km} \mathrm{s}^{-1}\right)\end{array}$ & $\begin{array}{r}\sigma_{R V_{1}^{\mathrm{Ca}}} \\
\left(\mathrm{km} \mathrm{s}^{-1}\right)\end{array}$ & $\begin{array}{r}R V_{2}^{\mathrm{Ca}} \\
\left(\mathrm{km} \mathrm{s}^{-1}\right)\end{array}$ & $\begin{array}{r}\sigma_{R V_{2}^{\mathrm{Ca}}} \\
\left(\mathrm{km} \mathrm{s}^{-1}\right)\end{array}$ \\
\hline 2450294.61180 & 80.888 & 1.174 & -139.721 & 1.304 & 99.409 & 1.822 & -109.541 & 1.838 \\
2450296.41523 & -158.761 & 1.600 & 114.952 & 1.585 & 0.000 & -999.999 & 0.000 & -999.999 \\
\hline
\end{tabular}

$\mathrm{O}-\mathrm{C}$ values is done with replacement, i.e. the same $\mathrm{O}-\mathrm{C}$ can be chosen several times while others are not used. With this artificial data set a new orbital fit is performed, leading to a slightly different set of parameters than the fit to the original data. Many artificial data sets are created and the error of a parameter is given by the standard deviation of that parameter from all the bootstrap solutions with respect to the parameter from the fit to the original data.

Four different solutions are given in Table 2. The first, solution 1, uses all the data and a forced circular fit; for such a close binary, tidal interactions should have circularized the orbit long ago. The resulting period is slightly shorter, but due to the error totally consistent with the period of 0.69809472 given by Keskin (1990) based on long-term photometry of ER Vul. In Table 2, we also give the parameters published by other authors. Our period is also consistent with that of Hill et al. (1990); however, they obtained significantly different periods from separate fits of the primary and secondary, which is not the case for our data. Our amplitudes seem to be significantly smaller, although the mass ratios obtained by us and by other authors are consistent.

The overall standard deviation $\sigma$ of an individual $R V$ from the fit is $3.7 \mathrm{~km} \mathrm{~s}^{-1}$, comparable to the one given by Hill et al. (1990). It should, however, be noted that Hill et al. (1990) used only $R V \mathrm{~s}$ in narrow phase intervals around maximum separation, while our $\sigma$ is based on all data, including the conjunction phases.

A similar fit has been performed, allowing for datasets obtained in different observing runs to have different $\gamma$ velocities, while all other orbital parameters are forced to be the same for all datasets. This was done in order to check for systematic errors in the wavelength zero points; different $\gamma$ velocities could, however, also result from an unknown third body in the system, which changes $\gamma$ of the short-period binary in a long-period orbit. Within the errors, all $\gamma$ velocities were the same, and the values for the other orbital parameters are indistinguishable from those given in Table 2. This shows that the wavelength calibration procedure worked well, and that there is no indication for a yet unknown third body in the system.

Solution 2 is based on data without the phases near conjunction. Phase intervals in which the secondary shows some systematic deviation from the curve of solution 1 (see below) are excluded. The overall $\sigma$ decreases significantly, particularly for the secondary due to the now missing systematics. All fit parameters are, within the combined errors of solutions 1 and 2, consistent; this means that the cut in phases is not necessary, and that the small systematic deviations of the secondary have no influence on the final orbital parameters. Therefore, in the following, always all data are used.

Solution 3 is a fit allowing for a non-zero eccentricity. As mentioned above, for physical reasons a circular orbit is expected. Yet, Northcott \& Bakos (1967) obtained a small (probably insignificant) eccentricity. Furthermore, the two stars in the ER Vul system are so close that the surfaces may already be significantly distorted, leading to a phase dependent difference between the centre of light (which is responsible for the measured $R V$ ) and the centre of mass; the orbital solution assumes that these two coincide. The phase dependent difference may thus result in a deformation of the $R V$ curve which could lead to an artificial eccentricity.

Indeed, solution 3 displays a nonzero eccentricity, about half of the eccentricity obtained by Northcott \& Bakos (1967). The longitudes of the periastron are very different. Our value of almost exactly $270^{\circ}$ indicates a curve whose shape is the closest to a pure cosine-curve for a given non-zero eccentricity. Furthermore, the value of the eccentricity that we obtain is less than $3 \sigma_{\mathrm{e}}$. Therefore, we conclude that the eccentricity is not real, i.e. the possible distortions of the stars do not lead to a deformation of the $R V$ curve which can be described by a nonzero eccentricity. We should, however, mention that the Lucy \& Sweeney $F$-test (Lucy \& Sweeney 1971; Lucy 1989) gave a probability of only $0.2 \%$ for a circular orbit, i.e. the reduction of $\chi^{2}$ is considered significant on the $99.8 \%$ level. It can be seen from Table 2 that all other orbital parameters are not significantly different for the elliptical or the circular orbit. We, therefore, adopt $e=0$.

\subsection{The improved orbital period of ER Vul}

Our dataset covers about 3 years, long compared to the orbital period. However, the longer the time base for period determination, the more accurate the resulting period. Usually, this fact is exploited by combining new $R V$ measurements with old ones from the literature. Given, however, that the old measurements for ER Vul were obtained in a way that leaves the suspicion of systematic errors (possibly responsible for the difference in the amplitudes measured here and in earlier papers) we did not perform an orbital fit to the combination of our $R V \mathrm{~s}$ with old $R V$ s. Instead, we used the fact that ER Vul is also an eclipsing binary. Many authors have measured eclipse times; these measured eclipse times can be compared with those predicted from our orbital solution. The $\mathrm{O}-\mathrm{C}$ vs. time will show a linear trend, if the real period is different from the one adopted in predicting the eclipse times. However, in such a close binary 
Table 2. The orbital solutions. Solution 1 is a fit of a circular orbit to all our data. Solution 2 is the same with phases around the conjunctions removed. Solution 3 uses again all data, but allows for a non-zero eccentricity. Solution 4 fixes the period to the improved value found from the eclipse times and fits a circular orbit to all data. All parameter errors are obtained from 10000 bootstrap runs. The three solutions presented in the literature are given for comparison.

\begin{tabular}{|c|c|c|c|c|c|c|c|}
\hline parameter & solution 1 & solution 2 & solution 3 & solution 4 & NB67 ${ }^{1}$ & $\operatorname{McL} 82^{1}$ & HFH90 ${ }^{1}$ \\
\hline$P$ (days) & $\begin{array}{r}0.69809401 \\
\pm 0.00000104\end{array}$ & $\begin{array}{r}0.69809369 \\
\pm 0.00000064\end{array}$ & $\begin{array}{r}0.69809395 \\
\pm 0.00000070\end{array}$ & $\begin{array}{r}0.69809458 \\
\pm 0.00000014\end{array}$ & 0.698084 & $\begin{array}{r}0.698082^{2} \\
\text { fixed }\end{array}$ & $\begin{array}{r}0.6980950 \\
\text { fixed }\end{array}$ \\
\hline$K_{1}\left(\mathrm{~km} \mathrm{~s}^{-1}\right)$ & $\begin{array}{r}135.20 \\
\pm 0.84\end{array}$ & $\begin{array}{r}135.02 \\
\pm 0.50\end{array}$ & $\begin{array}{r}135.40 \\
\pm 1.06\end{array}$ & $\begin{array}{r}135.20 \\
\pm 0.63\end{array}$ & $\begin{array}{r}138.5 \\
\pm 1.4\end{array}$ & $\begin{array}{r}136 \\
\pm 4\end{array}$ & $\begin{array}{r}139.5 \\
\pm 0.6\end{array}$ \\
\hline$K_{2}\left(\mathrm{~km} \mathrm{~s}^{-1}\right)$ & $\begin{array}{r}142.78 \\
\pm 1.04\end{array}$ & $\begin{array}{r}143.57 \\
\pm 0.34\end{array}$ & $\begin{array}{r}142.48 \\
\pm 1.15\end{array}$ & $\begin{array}{r}142.82 \\
\pm 0.76\end{array}$ & $\begin{array}{r}149.3 \\
\pm 1.4\end{array}$ & $\begin{array}{r}139 \\
\pm 2\end{array}$ & $\begin{array}{r}145.8 \\
\pm 0.5\end{array}$ \\
\hline$\gamma\left(\mathrm{km} \mathrm{s}^{-1}\right)$ & $\begin{array}{r}-25.51 \\
\pm 0.52\end{array}$ & $\begin{array}{r}-26.27 \\
\pm 0.36\end{array}$ & $\begin{array}{r}-25.64 \\
\pm 0.35\end{array}$ & $\begin{array}{r}-25.49 \\
\pm 0.39\end{array}$ & $\begin{array}{r}-25.2 \\
\pm 0.8\end{array}$ & $\begin{array}{r}-23 \\
\pm 3\end{array}$ & $\begin{array}{r}-24.6 \\
\pm 0.5\end{array}$ \\
\hline$e$ & $\begin{array}{r}0 \\
\text { fixed }\end{array}$ & $\begin{array}{r}0 \\
\text { fixed }\end{array}$ & $\begin{array}{r}0.0096 \\
\pm 0.0035\end{array}$ & $\begin{array}{r}0 \\
\text { fixed }\end{array}$ & $\begin{array}{r}0.017 \\
\pm 0.007\end{array}$ & $\begin{array}{r}0 \\
\text { fixed }\end{array}$ & $\begin{array}{r}0 \\
\text { fixed }\end{array}$ \\
\hline$\omega$ & - & - & $\begin{array}{l}270^{\circ} \\
\pm 21^{\circ}\end{array}$ & - & $\begin{array}{r}63^{\circ} \\
\pm 29^{\circ}\end{array}$ & - & - \\
\hline$T_{\text {peri }}(\mathrm{HJD})$ & - & - & $\begin{array}{r}2449726.263 \\
\pm 0.040\end{array}$ & - & $\begin{array}{r}2449726.303^{3} \\
\pm 0.001\end{array}$ & - & - \\
\hline$T_{\max }(\mathrm{HJD})$ & $\begin{array}{r}2449726.43711 \\
\pm 0.00058\end{array}$ & $\begin{array}{r}2449726.43785 \\
\pm 0.00036\end{array}$ & $\begin{array}{r}2449726.4346 \\
\pm 0.0012\end{array}$ & $\begin{array}{r}2449726.43703 \\
\pm 0.00043\end{array}$ & - & - & $\begin{array}{r}2449726.3883^{3} \\
\pm 0.0008\end{array}$ \\
\hline$T_{\text {conj }}(\mathrm{HJD})^{4}$ & $\begin{array}{r}2449726.61163 \\
\pm 0.00058\end{array}$ & $\begin{array}{r}2449726.61237 \\
\pm 0.00036\end{array}$ & $\begin{array}{r}2449726.6112 \\
\pm 0.0012\end{array}$ & $\begin{array}{r}2449726.61156 \\
\pm 0.00043\end{array}$ & - & $2449726.69420^{2,3}$ & - \\
\hline$P_{\text {rest }}$ (days) & $\begin{array}{r}0.6981534 \\
\pm 0.0000016\end{array}$ & $\begin{array}{r}0.6981549 \\
\pm 0.0000011\end{array}$ & $\begin{array}{r}0.6981537 \\
\pm 0.0000011\end{array}$ & $\begin{array}{r}0.69815395 \\
\pm 0.00000090\end{array}$ & - & - & - \\
\hline$a_{1} \sin i\left(R_{\odot}\right)$ & $\begin{array}{r}1.865 \\
\pm 0.012\end{array}$ & $\begin{array}{r}1.8624 \\
\pm 0.0069\end{array}$ & $\begin{array}{r}1.868 \\
\pm 0.015\end{array}$ & $\begin{array}{r}1.8650 \\
\pm 0.0087\end{array}$ & $\begin{array}{r}1.91 \\
\pm 0.02\end{array}$ & $\begin{array}{r}1.88 \\
\pm 0.06\end{array}$ & $\begin{array}{r}1.924 \\
\pm 0.009\end{array}$ \\
\hline$a_{2} \sin i\left(R_{\odot}\right)$ & $\begin{array}{r}1.969 \\
\pm 0.014\end{array}$ & $\begin{array}{r}1.9804 \\
\pm 0.0046\end{array}$ & $\begin{array}{r}1.965 \\
\pm 0.016\end{array}$ & $\begin{array}{r}1.970 \\
\pm 0.011\end{array}$ & $\begin{array}{r}2.05 \\
\pm 0.02\end{array}$ & $\begin{array}{r}1.93 \\
\pm 0.04\end{array}$ & $\begin{array}{r}2.012 \\
\pm 0.007\end{array}$ \\
\hline$m_{1} \sin ^{3} i\left(M_{\odot}\right)$ & $\begin{array}{r}0.798 \\
\pm 0.013\end{array}$ & $\begin{array}{r}0.8060 \\
\pm 0.0049\end{array}$ & $\begin{array}{r}0.796 \\
\pm 0.016\end{array}$ & $\begin{array}{r}0.7985 \\
\pm 0.0094\end{array}$ & $\begin{array}{r}0.89 \\
\pm 0.02\end{array}$ & $\begin{array}{r}0.76 \\
\pm 0.03\end{array}$ & $\begin{array}{r}0.860 \\
\pm 0.006\end{array}$ \\
\hline$m_{2} \sin ^{3} i\left(M_{\odot}\right)$ & $\begin{array}{r}0.756 \\
\pm 0.011\end{array}$ & $\begin{array}{r}0.7580 \\
\pm 0.0059\end{array}$ & $\begin{array}{r}0.756 \\
\pm 0.015\end{array}$ & $\begin{array}{r}0.7559 \\
\pm 0.0081\end{array}$ & $\begin{array}{r}0.83 \\
\pm 0.02\end{array}$ & $\begin{array}{r}0.75 \\
\pm 0.04\end{array}$ & $\begin{array}{r}0.823 \\
\pm 0.006\end{array}$ \\
\hline$q$ & $\begin{array}{r}0.9470 \\
\pm 0.0091\end{array}$ & $\begin{array}{r}0.9404 \\
\pm 0.0040\end{array}$ & $\begin{array}{r}0.9503 \\
\pm 0.0062\end{array}$ & $\begin{array}{r}0.9467 \\
\pm 0.0067\end{array}$ & $\begin{array}{r}0.93 \\
\pm 0.02\end{array}$ & $\begin{array}{r}0.98 \\
\pm 0.05\end{array}$ & $\begin{array}{r}0.957 \\
\pm 0.006\end{array}$ \\
\hline $\begin{array}{l}\sigma\left(\mathrm{km} \mathrm{s}^{-1}\right) \\
\sigma_{1}\left(\mathrm{~km} \mathrm{~s}^{-1}\right) \\
\sigma_{2}\left(\mathrm{~km} \mathrm{~s}^{-1}\right)\end{array}$ & $\begin{array}{l}3.70 \\
3.80 \\
3.70\end{array}$ & $\begin{array}{l}2.35 \\
3.13 \\
1.94\end{array}$ & $\begin{array}{l}3.63 \\
3.78 \\
3.61\end{array}$ & $\begin{array}{l}3.70 \\
3.75 \\
3.72\end{array}$ & $\begin{array}{l}- \\
-\end{array}$ & $\begin{array}{l}- \\
-\end{array}$ & $\begin{array}{r}3.03 \\
-\end{array}$ \\
\hline
\end{tabular}

${ }^{1}$ References: NB67 = Northcott \& Bakos (1967), McL82 = McLean (1982), HFH90 = Hill et al. (1990).

${ }^{2}$ Taken from Al-Naimiy (1978).

${ }^{3}$ Time advanced using their time and period.

${ }^{4}$ Time of eclipse with the primary in the back.

system, one also has to wonder whether the period is actually constant or systematically changing on long timescales. In that case, $\mathrm{O}-\mathrm{C}$ vs. time will show a parabolic trend.

We used all 80 eclipse times (or conjunction times from $R V$-measurements) that could be found in the literature. This includes our own conjunction times from separate orbital solutions to the individual datasets from different observing runs. In these fits, the period and all other orbital parameters were fixed to the values of solution 1 , except for the time $T_{0}$. The resulting conjunction times were assigned to the weighted average observing time of the run. The other eclipse or conjunction times were taken from: Northcott \& Bakos (1967), Battistini et al. (1974), Al-Naimiy (1978), Aslan et al. (1981), Pohl et al. (1982), İbanoğlu et al. (1985), Pohl et al. (1985), Milano et al. (1986), Pohl et al. (1987), Menella (1990), Srivastava et al. (1991), Wunder et al. (1992), Zeinali et al. (1995), Pribulla et al. (1999). In many cases, the authors list different times for the same minimum obtained in different filters. These were then averaged; if individual errors are available, the average is weighted. The error of the final minimum time is the mean error of the (weighted) mean. In a few cases, single values without any errors are given. Then, reasonable looking errors (like 0.0010 or 0.0005 , depending on our subjective judgement of the quality, helped by values given in the publication) were adopted.

The minimum and conjunction times are plotted in Fig. 2. Also shown are the results of a weighted line and parabola fit, where each point is weighted with the inverse variance based on its error. From the line fit, we conclude that the improved value for the period of ER Vul is

$P_{\text {obs }}^{\text {orb }}=0.69809458 \pm 0.00000014$, 


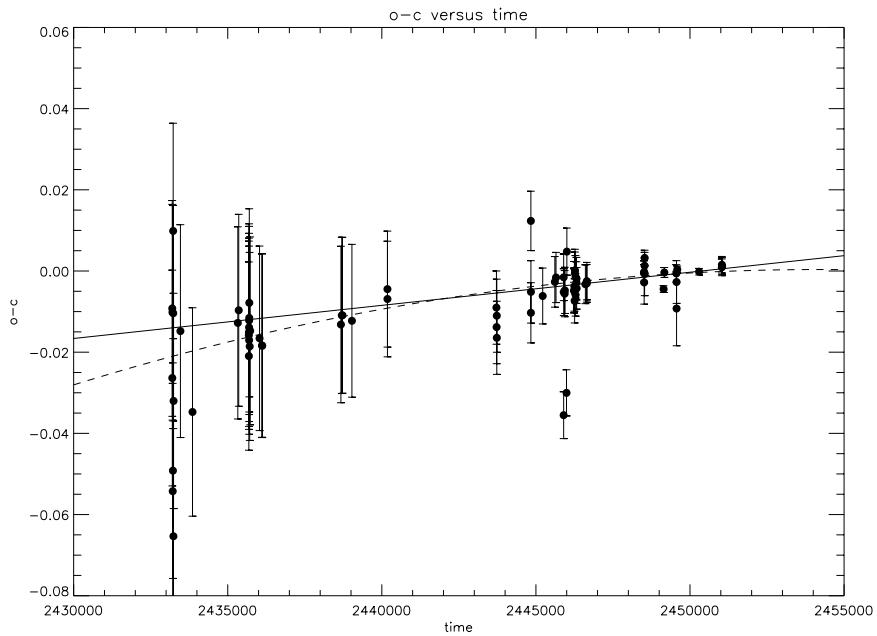

Fig. 2. $\mathrm{O}-\mathrm{C}$ values and their errors of the published minimum or conjunction times of ER Vul vs. time (see text for references). The period and conjunction time adopted to predict the minimum times are those from solution 1 in Table 2. A line (full line) and a parabolic fit (dashed line) are also shown.

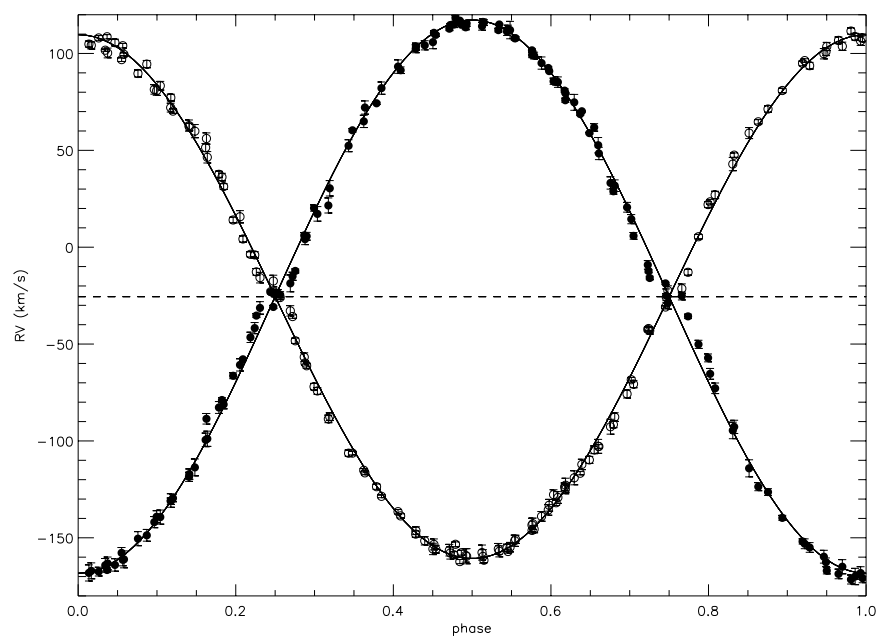

Fig. 3. All measured $R V$ s together with solution 4 from Table 2. Open circles: $R V_{1}$, filled circles: $R V_{2}$; in most cases, the errorbars are smaller than the symbol size.

still consistent, but slightly shorter than the period given by Keskin (1990), slightly longer than that of solution 1, but with an error 7 times smaller.

The quadratic fit gives formally a period $P_{\mathrm{obs}}(\mathrm{JD}=$ 2440000.0) $=0.69809498 \pm 0.00000029$ and a period change $\dot{P}=-(3.4 \pm 2.2) \times 10^{-11}$. The value of $\dot{P}$ is only 1.5 times its error; from Fig. 2 it is clear that the parabola and the line are not significantly different. Indeed, the reduced $\chi^{2}$ of the parabola fit is actually slightly larger than that of the line fit. We therefore conclude that the period given in Eq. (2) is the best available period for ER Vul at present, and that there is no evidence for a period change over at least 60 years.

Solution 4 in Table 2 is therefore the final orbital solution that we adopt. It fixes the period to the value given in Eq. (2) and the eccentricity to 0 . As one can see, solutions 4 and 1

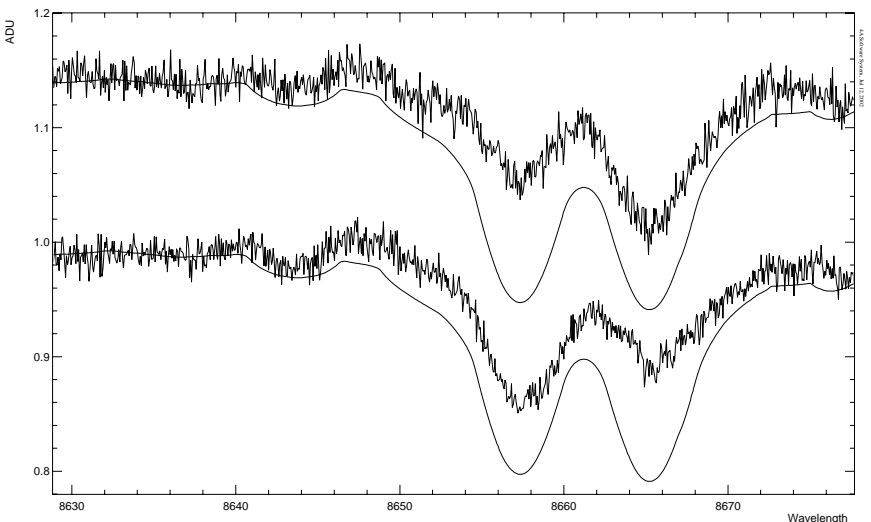

Fig. 4. The region around the Ca II IRT-line at $8662 \AA$. The noisy spectra are those of ER Vul (top at phase near 0.0, bottom at phase near 0.5 ), the smooth ones are those of the templates (a composite of two solar spectra, spun-up to $97 \mathrm{~km} \mathrm{~s}^{-1}$, with $\Delta R V=280 \mathrm{~km} \mathrm{~s}^{-1}$ ). For clarity, the spectra at phase 0.0 have been shifted upward by 0.15 .

are virtually identical. All $R V_{1}, R V_{2}$ values and their errors are shown together with the final orbital fit in Fig. 3.

As can be seen in Fig. 3, the secondary shows systematic deviations from the fit in a wide phase interval around the conjunctions. These can be attributed to a too large $v \sin i$ used for the secondary in the measurements. When the lines of the two stars are heavily blended, the cross-correlation will determine the $R V$ of each star based on the position of the red and (for the other star) blue wing of the blended feature. A too large width of the corresponding line in the template will therefore result in a too small $\triangle R V$, which is what we see in Fig. 3. We must therefore conclude that the secondary has $v \sin i<97 \mathrm{~km} \mathrm{~s}^{-1}$. From the deviations, which are in the extreme about $10 \mathrm{~km} \mathrm{~s}^{-1}$ larger than the noise around the curve near the maximum separation phases, we can conclude that $v \sin i$ of the secondary should not be smaller than about $87 \mathrm{~km} \mathrm{~s}^{-1}$; this is still significantly larger than the $71 \mathrm{~km} \mathrm{~s}^{-1}$, which Hill et al. (1990) determined for the secondary. It is remarkable that the primary does not show any significant systematic deviation from the curve near the conjunction phases. This must mean that $v \sin i=97 \mathrm{~km} \mathrm{~s}^{-1}$ is a good value for the primary.

\subsection{The radial velocities of Ca II $8662 \AA$}

One order is dominated by the Ca II IRT-line at $8662 \AA$; its $R V \mathrm{~s}$ deviate systematically from those of the other orders and are discussed therefore separately.

The CaII IRT is sensitive to chromospheric activity. Figure 4 compares the ER Vul spectra at phases near maximum separation with the simulated spectra (obtained in the same way as above by spinning up, shifting and averaging two solar spectra). As can be seen, the lines in the simulated spectra are much deeper than in ER Vul; the blue end shows that this cannot be due to a large error in the continuum normalization. The difference could be attributed to chromospheric emission in ER Vul. If this interpretation is correct, then the fact that the secondary (the blue component at the top) shows weaker absorption should mean that it has the stronger emission and 
is therefore more active than the primary. The weaker absorption for the secondary is a persistent feature, although at some times the line depths of primary and secondary are nearly equal. Figure 4 also implies that there is chromospheric emission not only in the two stars but more extended, even between the two stars and possibly extending also further out.

It is interesting to see the behaviour of the Ca II $R V$ s compared to the ones obtained from the other lines. Figure 5 shows our measurements compared to solution 4 in Table 2 (full line) and to a fit to the Ca II lines alone, where we limited the data used to the phase ranges around the extrema of the curve (dashed line). Note the much stronger systematic deviations of both stars from the curves, which cannot result from the above discussed effect, our overestimate of $v \sin i$ of the secondary. The orbital fit to the Ca II lines results in a more positive $\gamma$ of $-20.1 \mathrm{~km} \mathrm{~s}^{-1}$, and in $K_{1}=139.9 \mathrm{~km} \mathrm{~s}^{-1}, K_{2}=146.2 \mathrm{~km} \mathrm{~s}^{-1}$, i.e. $\gamma$ has increased by about $5 \mathrm{~km} \mathrm{~s}^{-1}$ and both amplitudes have increased as well. It is the increase of $\gamma$, which is not easy to understand in the binary system; the centre of mass should be the same, even if the Ca II emission is more extended, unless there is an asymmetric flow. On the other hand, Fig. 5 shows that the blue extrema of the two $R V$ curves are identical to those obtained from the other lines. Therefore, instead of assuming an increase of the amplitudes combined with a change of $\gamma$ one could also interpret the different behaviour as due to an asymmetry in the observed profile only visible near the red extrema of the curve. This means that there is a phase dependent asymmetry in the Ca II emission which shifts the $R V$ toward more positive velocities by about $10 \mathrm{~km} \mathrm{~s}^{-1}$ for each of the two stars, when that star is receding from us. This could mean a localized excess emission near the substellar point on the disk of that star or an excess absorption (weakening of the emission) at the point opposite to the substellar point. It cannot be the substellar point or the opposite exactly, because that would also be visible 0.5 orbits later. It is remarkable that this behaviour is persistent (with little noise) over the full 3 years of our data and that both stars show exactly the same phenomenon, despite a clear difference in activity level. Some of these findings are consistent with the results by Gunn \& Doyle (1997) who found the secondary to show stronger emission, and for the $\mathrm{H} \alpha$ line a velocity shift (in their limited phase interval near conjunction) of about $10 \mathrm{~km} \mathrm{~s}^{-1}$.

\section{Conclusions}

In order to measure composite, blended radial velocities $(R V \mathrm{~s})$ of binaries, we have developed a two-dimensional crosscorrelation method. It is based on a usual cross-correlation algorithm, as is widely available; it uses a grid of templates and optimizes via interpolation in the grid the $R V$ of one of the stars and the velocity difference $\Delta R V$ between the stars.

This technique is applied to a large number of highresolution spectra of the short-period RS CVn star ER Vul, an eclipsing binary with a period of about 0.7 . The large rotational velocity of the two solar type components lead to severe blending of the spectral features at nearly all phases.

In order to obtain proper templates, we determined, also based on cross-correlations, the rotational velocities of the two

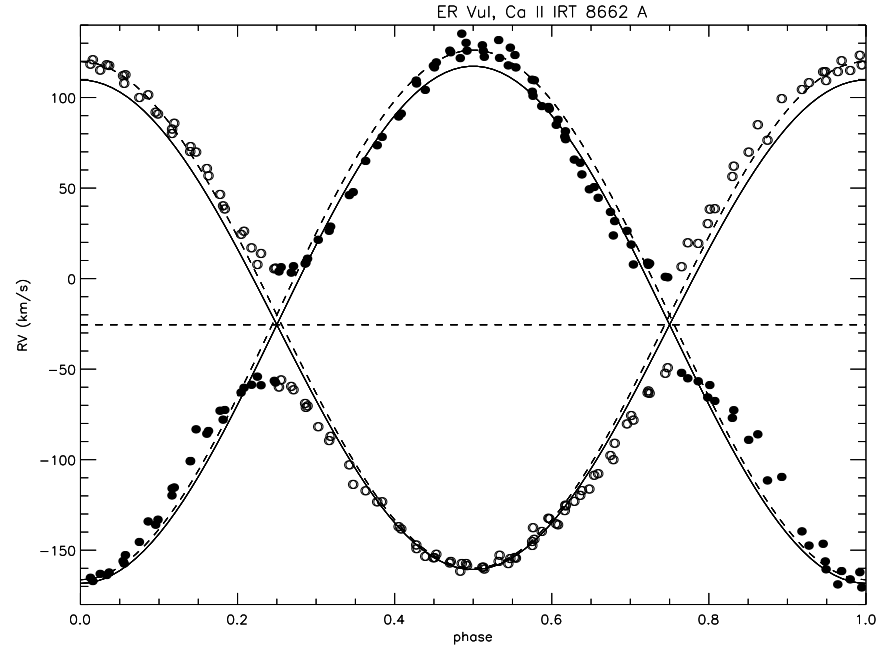

Fig. 5. The radial velocities of the order dominated by the Ca II IRT line at $8662 \AA$, compared to solution 4 of Table 2 (full line) and to an independent fit to the Ca II $R V \mathrm{~s}$ near maximum separation (dashed line).

stars. We found the same values of $v \sin i=(97.0 \pm 1.1) \mathrm{km} \mathrm{s}^{-1}$ for both stars; later it became clear that the secondary actually rotates somewhat slower. It is shown, however, that both stars rotate significantly faster than was determined by other authors.

The templates for the two-dimensional cross-correlation are constructed by artificially spinning up solar spectra, shifting them by $\triangle R V$, and average them to artificial composites. This is possible, because both components of ER Vul resemble closely the Sun. Most of the data consist of échelle spectra, in which several orders are suitable for $R V$ measurements; the results of the individual orders are averaged to give the final $R V \mathrm{~s} .137 R V \mathrm{~s}$ of both components of ER Vul are obtained. Combining our $R V \mathrm{~s}$ with conjunction and eclipse times from the literature, an improved binary period of $0.69809458 \pm$ 0 d 00000014 is obtained. With this, our $R V \mathrm{~s}$ result in a set of orbital parameters, which is consistent with most of the earlier investigations, except that the amplitudes $K_{1}, K_{2}$ seem to be significantly smaller; the mass-ratio, however, is nearly the same as obtained by other authors. It is shown in Fig. 3 that, apart from the small systematics caused by the over-estimate of the secondary's $v \sin i$, the $R V \mathrm{~s}$ of all, even heavily blended phases can be used.

We find that the Ca II IRT-line at $8662 \AA$ behaves systematically different from the other lines. For any of the two components, the $R V$ of $\mathrm{Ca}$ II is about $10 \mathrm{~km} \mathrm{~s}^{-1}$ larger, when the star recedes from us, but is consistent with the measurements from other lines when the star approaches us. Since the lines are much weaker than those in the solar-type templates, this is attributed to asymmetric chromospheric emission. While the emission is present always and is even originating between the two stars, the asymmetry affects only positive $R V \mathrm{~s}$. At most times, the secondary shows weaker $\mathrm{Ca}$ II absorption, which in this interpretation means that the emission is stronger, i.e. the secondary is the more active component; this has also been found by e.g. Gunn \& Doyle (1997). 
With the set of orbital parameters, which is given by solution 4 in Table 2, and the inclination obtained by earlier photometric modeling of the system, it is possible to derive constraints on other physical parameters of the system.

The inclination has been determined by several authors to cover the range $65^{\circ}<i<72^{\circ}$; the best values given by Hill et al. (1990) and İbanoğlu et al. (1993) seem to be consistent with $i=67^{\circ} \pm 1^{\circ}$, which is adopted in the following.

With the minimum masses obtained from the orbital solution and this inclination the two masses become

$m_{1}=(1.024 \pm 0.026) M_{\odot}, m_{2}=(0.969 \pm 0.024) M_{\odot}$.

When interpolating in the table of masses vs. spectral type given by Schmidt-Kaler (1982), these masses correspond to spectral types of G1 V+G3 V. Gray (1992, App. B) has a massspectral type relation in which G4 V has $1.00 M_{\odot}$; shifting the scale so that $\mathrm{G} 2 \mathrm{~V}$ obtains $1.00 M_{\odot}$ leads to spectral types for ER Vul of G1-2 V+G3 V. These types are consistent with those given by Hill et al. (1990) and Guinan et al. (1991), but indicate a larger similarity of the two components than in the still most often cited classification of G0 V+G5 V given by Northcott \& Bakos (1967). The types also support the suitability of the Sun as a template for the $R V$ measurements. The masses are slightly smaller than the ones given by Hill et al. (1990), whose mass ratio, however, is close to ours.

In such a close binary as ER Vul, it is very likely that tidal interactions have led to circularization of the orbit (which we confirm from our orbital solution) and synchronization of orbit and rotation. Assuming this, we adopt for the rotation period the orbital one (measured in the restframe of the system) and for the inclination of the rotation axes the above value of $i$. With this assumption and the measured value for $v \sin i$, which we show to be good for the primary (but not for the secondary), we obtain for the radius of the primary

$R_{1}=(1.454 \pm 0.019) R_{\odot}$,

substantially larger than the radii of $1.07 R_{\odot}$ given by Hill et al. (1990) and $1.17 R_{\odot}$ by İbanoğlu et al. (1993). With a radius of $R_{1}=1.17 R_{\odot}$ and the same assumptions as above, the primary should have $v \sin i=78 \mathrm{~km} \mathrm{~s}^{-1}$, a value even smaller than the one determined by Hill et al. (1990) and incompatible with our spectra. This would mean that the photometrically determined radii and our spectroscopically determined $v \sin i$ can only be consistent, if the rotation is not synchronous and/or the inclination of the rotation axis and that of the orbital axis are not equal; either of these seems difficult to understand. Note that Hill et al. (1990) obtain substantially different $v \sin i$ values for the two stars, yet the same radii, implying nonsynchronicity at least for one component, even if the axes are aligned.

With the inclination and the values $a_{1} \sin i, a_{2} \sin i$ and the mass ratio from the orbital solution we can use the approximation formula given by Eggleton (1983) to compute the effective radii $R^{R}$ of the Roche lobes of the two components, i.e. the radii of the spheres having the same volumes as the real Roche lobes. We obtain:

$R_{1}^{\mathrm{R}}=(1.598 \pm 0.010) R_{\odot}, R_{2}^{\mathrm{R}}=(1.559 \pm 0.010) R_{\odot}$.
This means that even with our large radius, the system is still detached; it also means that the radii presented by other authors would lead to even stronger detachment of the two stars. This seems to be incompatible with the photometric solutions for ER Vul (see e.g. Edalati \& Zeinali 1995, their Fig. 4), which seem to need a strong deformation of the two stars and therefore a large filling factor of the Roche lobe. In this context, it is important to note that the Roche lobe radius only depends on the separation of the two stars and the mass ratio, values which are similar in our orbital solution as in the orbital solutions presented by other authors.

The present $R V$ curve solution gives a good representation of the orbital shifts of the spectral lines, which can therefore be removed with great confidence prior to surface imaging. Preliminary surface imaging by Piskunov et al. (2001) based on the method described by Vincent et al. (1993) showed large cool areas on both components, which vary from season to season, as well as persistent bright areas in the substellar points, attributed to the reflection effect.

Acknowledgements. We are grateful to the referee G. Torres for a number of useful remarks which essentially improved the contents. FF and CD were enabled to stay at the Astronomy Division, University of Oulu, through an ERASMUS exchange from the University Paris XI Orsay. We made use of the SIMBAD database maintained at the CDS, Strasbourg, France.

\section{References}

Al-Naimiy, H. M. 1978, Ap\&SS, 53, 181

Aslan, Z., Asir, A., Engin, S., Tufekcioglu, Z., \& Yilmaz, N. 1981, IBVS, 1908

Battistini, P., Bonifazi, A., \& Guarnieri, A. 1974, IBVS, 951

Duemmler, R., Ilyin, I. V., \& Tuominen, I. 1997, A\&AS, 123, 209

Edalati, M. T., \& Zeinali, F. 1995, Ap\&SS, 234, 271

Efron, B., \& Tibshirani, R. J. 1993, An Introduction to the Bootstrap (New York: Chapman \& Hall)

Eggleton, P. P. 1983, ApJ, 268, 368

Gray, D. F. 1992, The observation and analysis of stellar photospheres, 2nd edn. (Cambridge: Cambridge Univ. Press)

Guinan, E. F., Bradstreet, D. H., Etzel, P. B., et al. 1991, BAAS, 23, 1412

Gunn, A. G., \& Doyle, J. G. 1997, A\&A, 318, 60

Gunn, A. G., Hall, J. C., Lockwood, G. W., \& Doyle, J. G. 1996, A\&A, 305,146

Hall, D. S. 1976, in Multiple Periodic Variable Stars, ed. W. S. Fitch, Proc. IAU Colloq 29 (Dordrecht: Reidel), 287

Hill, G., Fisher, W. A., \& Holmgren, D. 1990, A\&A, 238, 145

Huisong, T., \& Xuefu, L. 1987, A\&A, 172, 74

İbanoğlu, C., Akan, M. C., Evren, S., \& Tunca, Z. 1985, IBVS, 2782

İbanoğlu, C, Evren, S., Aken, M. C., Tunca, Z., \& Keskin, V. 1993, A\&A, 269, 310

Ilyin, I. V. 2000, Ph.D. Thesis, University of Oulu, Finland

Ilyin, I. V., \& Duemmler, R. 1999, in Precise Stellar Radial Velocities, Proc. IAU Coll. 170, ed. J. B. Hearnshaw, \& C. D. Scarfe, Astronomical Society of the Pacific, San Francisco, ASP Conf. Ser., 185, 63,

Keskin, V. 1990, Ap\&SS, 169, 165

Kurucz, R. L., Furenlid, I., Brault, J., \& Testerman, L. 1984, Solar Flux Atlas from 296 to $1300 \mathrm{~nm}$, National Solar Observatory Atlas No. 1 (on-line available at ftp://ftp.noao.edu/fts/fluxatl/) 
Lucy, L. B. 1989, Observatory, 109, 100

Lucy, L. B., \& Sweeney, M. A. 1971, AJ, 76, 544

McLean, B. J. 1982, MNRAS, 201,421

Menella, V. 1990, A\&A, 234, 203

Milano, L., Mancuso, S., Vittone, A., D’Orsi, A., \& Marcozzi, S. 1986, Ap\&SS, 124, 83

Northcott, R. J., \& Bakos, G. A. 1967, AJ, 72, 89

Piskunov, N., Vincent, A., Duemmler, R., Ilyin, I., \& Tuominen, I. 2001, in The 11th Cambridge Workshop on Cool Stars, Stellar Systems, and the Sun, ed. R. J. García López, R. Rebolo, \& M. R. Zapatero Osorio, ASP Conf. Ser., 223, CD-1285

Pohl, E., Evren, S., Tumer, O., \& Sezer, C. 1982, IBVS, 2189

Pohl, E., Tunca, Z., Gulmen, O., \& Evren, S. 1985, IBVS, 2793

Pohl, E., Akan, M. C., İbanoğlu, C., Sezer, C., \& Gudur, N. 1987, IBVS, 3078
Pribulla, T., Chochol, D., \& Parimucha, S. 1999, IBVS, 4751

Schmidt-Kaler, Th. 1982, in Landolt-Börnstein, Numerical Data and Functional Relationships in Science and Technology, New Series, Group VI: Astronomy, Astrophysics and Space Research, vol. 2b (Berlin: Springer), 30, Table 21

Srivastava, R. K., Padalla, T. D., \& Srivastava, J. B. 1991, Ap\&SS, 182,281

Tuominen, I., Ilyin, I., \& Petrov, P. 1999, in Astrophysics with the NOT, ed. H. Karttunen, \& V. Piirola, Univ. Turku, Finland, 47 Vincent, A., Piskunov, N., \& Tuominen, I. 1993, A\&A, 278, 523 Wunder, E., Wieck, M., Kilinc, B., et al. 1992, IBVS, 3760 Zeinali, F., Edalati, M. T., \& Mirtorabi, M. T. 1995, IBVS, 4190 Zucker, S., \& Mazeh, T. 1994, ApJ, 420, 806 